\title{
Estudo longitudinal sobre qualidade \\ e equidade no ensino fundamental brasileiro: GERES 2005
}

- Creso Franco*

Nigel Brooke ${ }^{* *}$

- Fátima Alves ${ }^{* * *}$

\section{Resumo}

Este artigo apresenta o relato do desenho metodológico do "Estudo longitudinal sobre qualidade e eficácia no ensino fundamental brasileiro: GERES 2005". O GERES ainda está em desenvolvimento e sua principal característica é o acompanhamento de uma mesma amostra de alunos dos anos iniciais do ensino fundamental ao longo de quatro anos. Cinco cidades participam do estudo, a saber: Belo Horizonte (MG), Rio de Janeiro (RJ), Salvador (BA), Campinas (SP) e Campo Grande (MS). O estudo começou em 2005 e envolve a aplicação de testes cognitivos em Leitura e em Matemática, bem como questionários contextuais para alunos, professores e diretores das escolas participantes. A Teoria de Resposta ao Item Paramétrica tem sido utilizada com o propósito de equalização das escalas para os diferentes níveis de escolarização (anos ou seus equivalentes), de modo que seja possível obter uma curva de crescimento da proficiência ao longo do tempo para cada aluno observado. O GERES tem como objetivo central investigar quais práticas pedagógicas e condições escolares contribuem para a promoção da eficácia e da eqüidade escolar, tendo como principal abordagem analítica a utilização de modelos multiníveis.

Palavras-chave: Estudo longitudinal. Avaliação educacional. Eficácia e eqüidade escolar. Modelos multiníveis.

\section{Longitudinal Study of Quality and Equity in the Brazilian Elementary Education: GERES 2005 Abstract}

This article presents a description of the methodology of "A longitudinal study of quality and equity in brazilian Elementary Education: GERES 2005". The GERES study is still being developed and its principal characteristic is the monitoring of a group of pupils throughout their first four years of Elementary Education. Five cities

* Doutor em Educação, University of Reading, Inglaterra; Professor do Departamento de Educação da Pontifícia Universidade Católica do Rio de Janeiro (PUC-Rio).E-mail: creso@puc-rio.br

** Doutor em Estudos do Desenvolvimento, Universidade de Sussex, Inglaterra; Professor convidado da Faculdade de Educação, Universidade Federal de Minas Gerais (UFMG).E-mail: n.brooke@terra.com.br

*** Doutora em Educação; Professora,PUC-Rio.E-mail: fcalves@puc-rio.br 
are taking part in the study: Belo Horizonte (MG), Rio de Janeiro (RJ), Salvador (BA), Campinas (SP) and Campo Grande (MS). The study started in 2005 and it involves the application of Reading and Math tests, as well as the application of context questionnaires to pupils, teachers and the principals of schools which take part in project. Item Response Theory has been used to equalize the scales for the different periods of schooling (years or their equivalent) in order to obtain a proficiency growth curve for each pupil for the period of the study. The GERES project aims to investigate those classroom activities and school conditions that contribute to the promotion of school efficiency and equity, having the use of multileveled models as analytical approach.

Keywords: Longitudinal study. Educational assessment. School efficacy and equity. Multileveled models.

\section{Estudio longitudinal sobre cualidad y equidad en la enseñanza fundamental brasileña: GERES 2005 Resumen}

Este artículo presenta relato del diseño metodológico del "Estudio longitudinal sobre cualidad y eficacia en la enseñanza fundamental brasileña: GERES 2005". EI GERES aún está en desenvolvimiento y su principal característica es el acompañamiento de una misma muestra de alumnos de los años iniciales de la enseñanza fundamental al longo de cuatro años. Cinco ciudades participan del estudio, a saber: Belo Horizonte (MG), Rio de Janeiro (RJ), Salvador (BA), Campinas (SP) e Campo Grande (MS). El estudio comenzó en 2005 y envuelve la aplicación de testes cognitivos en Lectura y en Matemática, bien como cuestionarios contextuales para alumnos, profesores y directores de las escuelas participantes. La Teoría de Respuesta al Ítem Paramétrica tiene sido utilizada con el propósito de ecualización de las escalas para los diferentes niveles de escolarización (años o sus equivalentes), de modo que sea posible obtener una curva de crecimiento de la proficiencia al longo del tiempo para cada alumno observado. El GERES tiene como objetivo central investigar cuales prácticas pedagógicas y condiciones escolares contribuyen para la promoción de la eficacia y de la equidad escolar, teniendo como principal abordaje analítico la utilización de modelos multiniveles.

Palabras clave: Estudio longitudinal. Evaluación educacional. Eficacia y equidad escolar. Modelos multiniveles.

\section{Contexto do início do GERES}

No espaço de pouco mais de uma década e meia, o Brasil criou um conjunto sofisticado de instrumentos para a avaliação do desempenho de alunos em todos os níveis de ensino. Especialmente para a educação básica, em âmbito nacional há o Sistema de Avaliação da Educação Básica que, atualmente é composto pelo SAEB e pela Prova Brasil. Destacam-se também as diversas experiências estaduais e municipais de avaliação. Todos esses projetos de avaliação têm o mérito de monitorar a situação educacional mediante uma seqüência de exercícios que, com métrica comparável ao longo do tempo, apresentam as tendências na evolução da qualidade da 
educação brasileira. Por isso, pode-se afirmar que a demanda por informações sobre o estado e a evolução da qualidade da educação brasileira tem sido satisfeita pelos projetos de avaliação em curso.

O interesse dos pesquisadores e gestores de sistemas educacionais consiste em conhecer o efeito de políticas e práticas educacionais sobre o aprendizado dos alunos. No entanto, as mencionadas experiências de avaliação da educação não oferecem os dados mais adequados para inferências causais acerca de quais políticas e práticas fazem diferença em educação. Em outras palavras, enquanto uma seqüência de resultados do SAEB oferece boa orientação sobre a tendência da qualidade da educação nacional, ela não oferece a possibilidade de estudo pormenorizado sobre quais fatores promovem ou conspiram contra a qualidade. Veremos a razão disto em detalhes.

Os projetos de avaliação educacional em larga escala já consolidados no Brasil são surveys seccionais, isto é, em um dado momento mensuram o desempenho dos alunos e promovem um levantamento de informações sobre alunos e suas escolas. No entanto, o fato do SAEB ter coletado informações sobre alunos e escolas brasileiras em, por exemplo, 1995, não significa que esse survey pode servir para investigar quais fatores escolares estavam associados ao maior desempenho em leitura ou em matemática naquele ano. Empreendimentos desta natureza possuem uma limitação básica: a medida de desempenho em leitura ou em matemática é um agregado do aprendizado dos alunos ao longo de muitos anos. Já as medidas escolares disponíveis referem-se às condições escolares no ano da coleta de dados. Esta falta de sintonia temporal entre a medida do desempenho e as medidas das condições escolares, fragiliza as análises e inviabilizam a formulação de políticas de qualidade e eqüidade baseadas em evidências sólidas.

Diversos autores mostram as dificuldades da utilização de dados seccionais para investigar a relação entre fatores escolares e desempenho acadêmico. Além do problema em atribuir causalidade (RAUDENBUSH; FOTIU; CHEONG, 1998), a literatura enfatiza a ausência de medidas anteriores do desempenho dos alunos para calcular o ganho atribuível aos fatores escolares (GOLDSTEIN, 1995) e a impossibilidade de fazer inferências confiáveis sobre a eficácia das escolas com base em uma única medida do desempenho (GOLDSTEIN et al., 2000). Estas dificuldades levam vários autores a especificar certos requisitos mínimos para o estudo dos fatores escolares, entre os quais se destaca a necessidade da coleta repetida de dados em um desenho de pesquisa longitudinal. Lee (2001) explica que este desenho permite investigar, de fato, o aprendizado, que é uma medida de mudança no tempo, em vez de medir só o rendimento, que é um atributo estático. Goldstein (1997, p. 376, tradução nossa), por outro lado, estabelece quatro condições mínimas para formular inferências satisfatórias a respeito de uma determinada escola, tal como a sua melhoria ou não:

1. que o estudo seja longitudinal de modo que diferenças preexistentes entre os alunos e eventos contingentes subseqüentes entre as escolas possam ser levados em consideração;

2. que se incumbe da analise multinível apropriada para que as inferências estatísticas sejam validas e, em particular, que a "eficácia diferencial" seja investigada; 
3. que haja alguma replicação no tempo e espaço para comprovar a replicabilidade; e,

4. que se ofereça alguma explicação plausível do processo pelo qual as escolas se tornam mais eficazes.

Advertências como estas, formuladas a partir da década de 1990, tiveram grandes implicações na investigação sobre qualidade e eficácia escolar. Em muitos países, os esforços de avaliação deslocaram-se no sentido de contemplar estudos longitudinais. Observe-se que os autores apontaram as limitações dos estudos seccionais e assinalaram a necessidade de desenhos de pesquisa alternativos para a investigação do efeito de políticas e práticas escolares. Devemos mencionar que outros autores valorizam como uma a alternativa os estudos experimentais. Concordamos que tais estudos, quando operacional e eticamente viáveis, consistem na situação mais adequada para inferências causais. No entanto, o desenho de pesquisa do Estudo Longitudinal da Geração Escolar - GERES, não seguiu esta vertente por diversas razões. Dentre as mais relevantes estão:

- estudos experimentais precisam ter um foco bem delimitado, de modo que se possa definir precisamente o tratamento, a ser administrado ao grupo experimental, que deverá ser comparado ao grupo de controle. Como no Brasil ainda há poucas evidências sobre o que promove mais aprendizado dos alunos, um estudo com foco mais amplo, que possa avaliar um conjunto maior de políticas e práticas escolares pode dar maior contribuição em termos de produção de conhecimento e de desenvolvimento de políticas públicas baseadas em evidências;

- dificuldades operacionais costumam conspirar contra a validade externa de estudos experimentais. Em outras palavras, escolas, professores, pais de alunos e alunos que concordam em participar de estudo experimental, no qual o tratamento será alocado, a parte dos participantes de modo aleatório podem conformar um grupo tão peculiar que a generalização dos achados para o sistema educacional pode ser inadequada.

O GERES surgiu, então, em um contexto no qual a utilização de dados de avaliação ganha cada vez mais espaço na pesquisa e na formulação de políticas educacionais. $O$ GERES é conseqüência de um esforço colaborativo de diversos pesquisadores que nos últimos anos tem produzido estudos sobre qualidade, eqüidade e desigualdades de oportunidades educacionais. Diferentemente dos sistemas de avaliação dos estudantes em curso no Brasil, esta é uma pesquisa longitudinal, na qual uma mesma amostra de alunos é observada ao longo dos anos iniciais do Ensino Fundamental. Sem dúvida, a escolha por este desenho de pesquisa é uma contribuição para o conhecimento do que faz diferença na educação brasileira. $\bigcirc$ foco deste artigo é justamente apresentar os principais aspectos metodológicos que norteiam o desenvolvimento da pesquisa GERES.

\section{Objetivos do GERES}

Conforme apontado anteriormente, o GERES focaliza os anos iniciais do Ensino Fundamental e tem como principais objetivos: 
1. identificar as características escolares que maximizam a aprendizagem dos alunos e que minimizam o impacto da origem social sobre o aprendizado;

2. identificar os fatores escolares que diminuem a probabilidade de repetência dos alunos;

3. identificar aquelas características da escola que reduzem a probabilidade do absentismo.

\title{
Esquema geral da metodologia GERES
}

Como já enfatizada, a principal razão que nos leva a coletar dados primários, ao invés de simplesmente utilizar os dados dos grandes projetos de avaliação existentes no Brasil, é a natureza seccional dos dados dos grandes projetos de avaliação, o que não permite conclusões bem fundamentadas sobre os recursos e práticas escolares que promovem a aprendizagem. Um dos principais propósitos do GERES é justamente a obtenção de resultados que possam subsidiar políticas educacionais baseadas em evidências. Desta forma, este artigo enfoca dois elementos chaves da metodologia GERES: o desenho da pesquisa e a abordagem analítica. A este respeito, Lee (2001, p. 34, tradução nossa) mostra que:

\begin{abstract}
Publicações recentes sobre a avaliação educacional argumentam frequentemente que muitos estudos chegam a conclusões equivocadas porque os métodos [de análise] estão errados. Isto é particularmente grave quando são formuladas políticas sociais com base nesses estudos. Em geral, nas análises de um nível só, os efeitos dos fatores contextuais são sistematicamente subestimados. No entanto, muitas políticas educacionais são implementadas no nível do grupo - muitas vezes no nível da escola. Se quisermos saber se determinada política atingiu os objetivos estabelecidos, é vital analisar os dados da forma correta. Naturalmente, precisamos ter dados bons. Igualmente importante, precisamos de mais pesquisadores e os pesquisadores precisam estar familiarizados com, e saber usar, os métodos de pesquisa multinível.
\end{abstract}

Os argumentos apresentados apontam para o desenho longitudinal, no qual os mesmos alunos serão observados ao longo do processo de escolarização, e uma abordagem analítica multinível. Essas características envolvem relações com outros aspectos metodológicos, nomeadamente, a amostragem, os instrumentos cognitivos e os contextuais. Todos esses aspectos são apresentados a seguir.

\section{Desenho da pesquisa}

A pesquisa adota desenho longitudinal de painel, no qual a mesma amostra de escolas e de alunos será observada ao longo de quatro anos. $\bigcirc$ ano de 2004 foi dedicado à consolidação do suporte institucional do projeto' e ao desenvolvimento e pré-testagem dos instrumentos cognitivos e contextuais. A primeira onda de medida foi tomada em março de 2005, com a observação de alunos do $2^{\circ}$ ano do ensino fundamental ${ }^{2}$ (ou seu equivalente, quando a organização do ensino era em

1 Participam da pesquisa seis instituições de ensino superior: UFMG, PUC-Rio, Unicamp, UEMS, UFJF e UFBa .2 Antiga $1^{a}$ série do Ensino Fundamental. 
ciclos) e a segunda onda de medida ocorreu em novembro de 2005. O painel foi observado também em novembro de 2006 e 2007 e as observações serão concluídas em novembro de 2008, viabilizando o acompanhamento da amostra ao longo de quatro anos letivos. A pesquisa vem tomando todo cuidado possível para fazer a distinção entre 'valor agregado pela escola' e o efeito da 'seleção'. Em termos práticos, isso significa que alunos que não são aprovados continuam sendo observados desde que permaneçam na mesma escola ou se transfiram para escola da amostra. Como não seria viável continuar observando alunos que se transferem para escolas fora da amostra, estes alunos são perdidos. Cabe ressaltar que o cuidado de observar estes alunos não aprovados, bem como considerar os dados sobre os alunos evadidos e/ou transferidos, evitará que a análise confunda como 'valor agregado pela escola' aquilo que se deve unicamente à eventual exclusão da escola de alunos de desempenho menor.

\section{População e amostra}

Os objetivos da pesquisa exigem a estimação de modelos de regressão dentro das escolas. Além disto, nos anos iniciais do Ensino Fundamental o que ocorre dentro da sala de aula é particularmente importante, razão pela qual faz-se necessária a observação do maior número possível de salas de aula dentro de cada escola. Ainda que uma amostra probabilística de escolas públicas e privadas brasileiras fosse desejável, isto teria impacto financeiro e operacional que iria além das possibilidades da equipe de pesquisa, o que nos levou a concentrar a pesquisa em escolas de cinco cidades brasileiras. Cada uma das cidades foi considerada como um estrato e, dentro de cada cidade, foi selecionada uma amostra probabilística complexa de escolas, turmas e alunos a partir do cadastro do Censo Escolar de 2003, excluídas as escolas que não possuíam 10 alunos ou mais matriculados no $2^{\circ}$ ano do ensino fundamental (ou seu equivalente em ciclos) ${ }^{3}$.

A Tabela 1 resume o primeiro estágio da amostragem, relativo à seleção de escolas.

3 Para não incluir escolas muito grandes, no caso da rede privada foram incluídas somente escolas com três turmas ou menos no $2^{\circ}$ ano.

Ensaio: aval. pol. públ. Educ., Rio de Janeiro, v. 16, n. 61, p. 625-638, out./dez. 2008 


\begin{tabular}{|c|c|c|c|c|c|}
\hline Estrato & Cidade & \begin{tabular}{|c|} 
Dependência \\
Administrativa
\end{tabular} & $\begin{array}{l}\text { Tamanho } \\
\text { do Estrato }\end{array}$ & $\begin{array}{c}\text { Número de } \\
\text { Escolas } \\
\text { Selecionadas }\end{array}$ & $\begin{array}{c}\% \text { da } \\
\text { Amostra }\end{array}$ \\
\hline 1 & \multirow[t]{2}{*}{ Rio de Janeiro } & Municipal & 765 & 30 & 3,9 \\
\hline 2 & & Privada & 805 & 30 & 3,7 \\
\hline 3 & \multirow{3}{*}{ Belo Horizonte } & Estadual & 155 & 20 & 12,9 \\
\hline 4 & & Municipal & 135 & 20 & 14,8 \\
\hline 5 & & Privada & 144 & 20 & 13,9 \\
\hline 6 & \multirow{3}{*}{ Campinas } & Estadual & 95 & 20 & 21,1 \\
\hline 7 & & Municipal & 39 & 20 & 51,3 \\
\hline 8 & & Privada & 47 & 20 & 42,6 \\
\hline 9 & \multirow{3}{*}{ Campo Grande } & Estadual & 70 & 20 & 28,6 \\
\hline 10 & & Municipal & 76 & 19 & 26,3 \\
\hline 11 & & Privada & 80 & 20 & 25,0 \\
\hline 12 & \multirow{3}{*}{ Salvador } & Estadual ${ }^{*}$ & 67 & 21 & 31,3 \\
\hline 13 & & Municipal** $^{* *}$ & 332 & 20 & 6,0 \\
\hline 14 & & Particular & 278 & 20 & 7,2 \\
\hline 15 & \multicolumn{2}{|c|}{$\begin{array}{l}\text { Escolas Federais ou Colégios de } \\
\text { Aplicação de Universidades Públicas }\end{array}$} & 9 & 09 & 100,0 \\
\hline \multicolumn{3}{|c|}{ Total } & 3.097 & 310 & 10,0 \\
\hline
\end{tabular}

Tabela 1 - Critérios de estratificação e número de escolas por estrato. Fonte: os Autores (2008).

Objetivando maximizar a diversidade de escolas selecionadas, foi utilizado um critério adicional de estratificação, variável conforme a disponibilidade de informações em cada estrato. Idealmente, a variável de estratificação foi a medida socioeconômica média das escolas. Nos estratos nos quais esta variável não estava disponível utilizou-se como variável de estratificação o desempenho médio das escolas em avaliação censitária ou, na falta desta, medida de equipamento das escolas, construída a partir de dados do censo escolar.

Selecionadas as escolas, as turmas do $2^{\circ}$ ano e seus alunos foram selecionados com probabilidade 1 (isto é, todas as turmas do $2^{\circ}$ ano e seus alunos foram grupados). Este desenho objetivou o aumento da amostra dentro de cada escola e cada turma, para permitir maiores opções em relação ao estudo do efeito escola e efeito turma. A Tabela 2 resume as informações sobre o número de escolas, salas de aula e alunos selecionados em cada estrato.

* A amostra de escolas estaduais foi dividida entre escolas sem previsão de municipalização e escolas a serem municipalizadas em 2005/2006.

** A amostra de escolas municipais foi dividida entre escolas originalmente municipais e escolas municipalizadas entre 2002/2004. 


\begin{tabular}{|c|c|c|c|c|c|}
\hline Cidade & $\begin{array}{c}\text { Dependência } \\
\text { Administrativa }\end{array}$ & $\begin{array}{l}\text { Número de } \\
\text { Escolas }\end{array}$ & $\begin{array}{l}\text { Número de } \\
\text { Salas de } \\
\text { Aula }\end{array}$ & $\begin{array}{l}\text { Número de } \\
\text { Alunos }\end{array}$ & $\begin{array}{c}\text { Número de } \\
\text { Alunos } \\
\text { Testados } \\
\text { (Março 2005) }\end{array}$ \\
\hline \multirow[t]{2}{*}{ Rio de Janeiro } & Municipal & 30 & 90 & 2.527 & 2.362 \\
\hline & Privada & 30 & 55 & 1.032 & 985 \\
\hline \multirow{3}{*}{ Belo Horizonte } & Estadual & 20 & 64 & 1.682 & 1.617 \\
\hline & Municipal & 20 & 88 & 2.036 & 1.953 \\
\hline & Privada & 20 & 32 & 669 & 651 \\
\hline \multirow{3}{*}{ Campinas } & Estadual & 20 & 74 & 2.158 & 2.050 \\
\hline & Municipal & 20 & 73 & 1.919 & 1.753 \\
\hline & Privada & 20 & 42 & 804 & 790 \\
\hline \multirow{3}{*}{ Campo Grande } & Estadual & 20 & 38 & 845 & 838 \\
\hline & Municipal & 19 & 97 & 2.418 & 2.410 \\
\hline & Privada & 20 & 27 & 342 & 344 \\
\hline \multirow{3}{*}{ Salvador } & Estadual & 21 & 25 & 692 & 608 \\
\hline & Municipal & 20 & 113 & 3.032 & 2.406 \\
\hline & Privada & 20 & 30 & 544 & 516 \\
\hline \multicolumn{2}{|c|}{$\begin{array}{l}\text { Escolas Federais ou Colégios de } \\
\text { Aplicação de Universidades Públicas }\end{array}$} & 09 & 35 & 869 & 821 \\
\hline \multicolumn{2}{|c|}{ Total } & 310 & 883 & 21.569 & 20.104 \\
\hline
\end{tabular}

Tabela 2 - Tamanho da amostra de turmas e alunos, por estrato.

Fonte: os Autores (2008).

Na primeira onda da pesquisa (março de 2005), observaram-se todas as escolas e salas de aula e procurou-se testar todos os alunos selecionados. Alunos que faltaram no dia do teste não foram examinados em outros dias, mas continuaram na pesquisa e foram testados em ondas subseqüentes quando freqüentavam as escolas da amostra. Nas ondas subseqüentes, a pesquisa procurou testar todos os alunos inicialmente selecionados, independentes de promoção para ano seguinte. Alunos que foram transferidos de escolas não foram testados, exceto quando houve transferência em bloco de alunos de escolas, devido a medidas administrativas da rede de ensino, como no caso de alunos da rede municipal de Rio de Janeiro em $2007^{4}$. Nas ondas seguintes, a pesquisa continuou coletando medidas sobre a escola e sobre as salas de aula dos anos subseqüentes (mas não das salas de aula dos alunos repetentes, pois o número de alunos nessas salas de aula não era suficientemente grande para viabilizar análises nessas salas de aula). Alunos não selecionados ao início da pesquisa, mas que vieram a participar das turmas para as quais foram promovidos os alunos GERES, também foram testados.

\footnotetext{
4 Por decisão das autoridades municipais, aproximadamente 600 alunos GERES foram transferidos para outras escolas durante o ano escolar 2007. Além de seguir e reintegrar esses alunos à pesquisa, os seus novos colegas de turma também foram incorporados ao GERES.
} 
peso amostral compatível com o plano amostral original foi calculado para cada aluno, turma e escola para permitir que os dados GERES fossem interpretados como representativos das cinco cidades da pesquisa As informações que viabilizam o cálculo de peso amostral para situações diferenciadas, como aquela que envolve a inclusão dos alunos que passaram a ser testados por terem sido posteriormente alocados em turma sujeita a teste GERES, estão organizadas de modo a viabilizar ponderações alternativas.

\section{Instrumentos cognitivos}

Testes adequados aos anos iniciais do ensino fundamental foram preparados com base em matrizes de habilidades de Leitura e Matemática, preparados por especialistas de três das universidades participantes da pesquisa, a UFMG, a PUC-Rio e a UFJF. Os itens utilizados foram previamente testados em escolas públicas e privadas das cidades de Juiz de Fora e Rio de Janeiro. Em cada onda, todos os alunos presentes foram submetidos tanto ao teste de leitura quanto ao de matemática. De modo a maximizar a discriminação dos testes, foram preparadas duas versões em cada onda, uma mais fácil e outra mais difícil. As diferentes versões dos testes (dentro e entre ondas) possuíam itens comuns, de modo a viabilizar escores equalizados a partir da Teoria de Resposta ao Item (TRI). Na primeira onda, a definição da versão do teste a ser respondido pelos alunos foi feita a partir de informações prévias sobre as escolas em que estudavam. A partir da segunda onda, esta definição foi baseada nos resultados das ondas anteriores. A partir da quarta onda, os testes incorporaram itens do SAEB tanto de leitura quanto de matemática. A inclusão desses itens permite a equalização dos resultados dos alunos GERES com a população brasileira testada pelo SAEB.

Em consonância com as matrizes de leitura e matemática, foram desenvolvidas duas escalas para descrever o desempenho dos alunos em termos das habilidades em fase de consolidação e as que já foram dominadas. Estas escalas, indispensáveis para a comunicação dos resultados às escolas e a interpretação pedagógica do desempenho das turmas, permitem mostrar a proporção de alunos em cada faixa ou nível de desempenho e comparar as escolas e redes em termos dessas proporções. As proficiências dos alunos GERES são recalculadas a cada onda, o que permite a comparação das diferentes ondas. Todos os cálculos das proficiências em todas as ondas são feitos pela mesma equipe, usando a mesma metodologia, o que garante a confiabilidade dos resultados cognitivos obtidos.

\section{Instrumentos contextuais}

Informações sobre as escolas, salas de aula e famílias continuam sendo obtidas a partir de questionários administrados a diretores, professores, pais e alunos, além de instrumento de observação preenchido por aplicador externo à escola. O questionário dos pais e a primeira versão do questionário dos alunos, aplicado na $4^{a}$ onda, serviram para identificar o nível educacional e profissão dos pais e os objetos de consumo presentes na casa do aluno. Essas informa- 
ções foram utilizadas na confecção da variável do nível socioeconômico das famílias. O Quadro 1 resume os questionários utilizados em cada onda.

\begin{tabular}{|l|l|}
\hline Escola & $\begin{array}{l}\text { Aplicado em Março de 2005. Informações básicas sobre a infra-estrutura da } \\
\text { escola. }\end{array}$ \\
\hline Diretores & $\begin{array}{l}\text { Aplicado em Março de 2005 e em novembro de 2007. Informações sobre sua } \\
\text { experiência e formação. Sobre características do financiamento da escola } \\
\text { (anuidades, bolsas de estudo, etc) em caso de escola privada. Sobre processos } \\
\text { de nomeação do diretor (escolas públicas). Sobre relacionamento com comu- } \\
\text { nidade. Critérios de formação de turmas. A ser reaplicado em 2008. }\end{array}$ \\
\hline Professores & $\begin{array}{l}\text { Aplicado em março de 2005 e em todos os anos da pesquisa em novembro. } \\
\text { Previsão do professor sobre desempenho da turma nos testes. } \\
\text { Estilo e liderança do diretor. Práticas de sala de aula. Formação. Práticas } \\
\text { culturais. }\end{array}$ \\
\hline Pais & $\begin{array}{l}\text { Aplicado entre o segundo semestre de 2005 e o segundo semestre de 2007. } \\
\text { Caracterização sócio-demográfica da família. Aplicados aos pais de alunos } \\
\text { novos incorporados à pesquisa a partir de } 2007 .\end{array}$ \\
\hline Alunos & $\begin{array}{l}\text { Aplicado em novembro de } 2007 \text { (versão reduzida) e em novembro de 2008 } \\
\text { (versão ampliada). Caracterização sócio-demográfica da família. }\end{array}$ \\
\hline
\end{tabular}

Quadro 1 - Os questionários utilizados.

Fonte: os Autores (2008).

\section{Abordagem analítica}

A abordagem analítica é baseada nos modelos multiníveis. A investigação sobre eficácia e equidade escolar, que tem como variável dependente a proficiência dos alunos, estará baseada em modelos de regressão lineares multiníveis. No contexto de modelagem de curvas de crescimento (growth curve modeling), cada aluno terá sua própria curva de crescimento e será investigado como as características da sala de aula e da escola. Para os objetivos adicionais definidos anteriormente - características escolares que aumentam a probabilidade de promoção, que diminuem o absenteísmo - modelos lineares mostram-se inadequados, iá que a variável dependente possuirá distribuição não normal e não normalizável. Nestes casos, a abordagem será baseada em modelos multiníveis não lineares. Como o tipo de teoria de medida mais adequada à construção de escalas a partir de questionários contextuais é um tema ainda em aberto, a pesquisa comparará diferentes abordagens teóricas para a construção de escalas: TRI não paramétrica, TRI paramétrica e análise fatorial confirmatória, esta última no âmbito de modelagem por equações estruturais.

\section{Pesquisa com subamostra de pais}

No ano de 2007 foi desenvolvido uma pesquisa tipo survey junto a uma subamostra de pais de alunos das escolas GERES de Belo Horizonte para deter- 
minar a relação entre as condições e atitudes dos pais e o desempenho dos alunos. A população amostrada excluiu alunos do quintil superior de NSE, alunos com menos de duas participações nas avaliações do GERES e alunos de endereço desconhecido. A amostra de 300 alunos foi estratificada para incluir alunos da escola federal, escolas privadas, escolas estaduais e municipais de alto desempenho (quintil superior de Belo Horizonte) na Prova Brasil e das demais escolas estaduais e municipais. O questionário, desenvolvido pelo Observatório Sociológico Família-Escola, foi aplicado aos pais ou responsáveis dos alunos no segundo semestre de 2007.

\section{Relacionamento pesquisa-escola}

Fazem parte da metodologia da pesquisa as relações entre os pesquisadores e as escolas participantes e a manutenção dessas relações para o período da pesquisa. Em se tratando de um estudo de painel, torna-se vital que as escolas se sintam parceiras e recebam dos pesquisadores os incentivos necessários para manter o vinculo com o GERES até o final do processo de coleta de informações. A incorporação das escolas à pesquisa foi feita mediante declaração formal de adesão e, após cada onda de aplicação, elas recebem um relatório que permite identificar as faixas de desempenho dos alunos das diferentes turmas. Outras atividades com representantes das escolas, incluindo reuniões para explicar o conteúdo dos relatórios, variam de pólo a pólo. A coordenação central do GERES mantém uma página na Internet contendo informações, publicações e outros documentos da pesquisa e anualmente produz um boletim para circulação entre os membros das escolas participantes. 


\section{Cronograma da pesquisa GERES}

Quadro 2 apresenta o cronograma de aplicação dos instrumentos de pesquisa, bem como as etapas de desenvolvimento da pesquisa.

\begin{tabular}{|c|c|c|}
\hline Ano & Mês & Atividade/Instrumento \\
\hline 2004 & Novembro & Pré-teste instrumentos de leitura e matemática \\
\hline \multirow{8}{*}{2005} & \multirow{5}{*}{$\begin{array}{c}\text { Março } \\
\left(1^{a} \text { Onda) }\right.\end{array}$} & Questionário escola/recursos e equipamentos \\
\hline & & Questionário do diretor \\
\hline & & Testes de leitura e matemática \\
\hline & & Questionário do professor \\
\hline & & Levantamento sobre sala de aula \\
\hline & \multirow{3}{*}{$\begin{array}{l}\text { Novembro } \\
\left(2^{a} \text { Onda) }\right.\end{array}$} & Relatório de escola $1^{a}$ Onda \\
\hline & & Testes de leitura e matemática \\
\hline & & Questionário de pai ou responsável \\
\hline \multirow{6}{*}{2006} & Maio & Relatório de escola $2^{a}$ Onda \\
\hline & Junho & Pré-teste instrumentos de leitura e matemática \\
\hline & \multirow{4}{*}{$\begin{array}{l}\text { Novembro } \\
\left(3^{a} \text { Onda) }\right.\end{array}$} & Testes de leitura e matemática \\
\hline & & Questionário do professor \\
\hline & & Questionário do diretor \\
\hline & & Questionário de pai ou responsável \\
\hline \multirow{7}{*}{2007} & Maio & Relatório $3^{a}$ Onda \\
\hline & Junho & Pré-teste instrumentos de leitura e matemática \\
\hline & $\begin{array}{l}\text { Setembro- } \\
\text { dezembro }\end{array}$ & Pesquisa em subamostra de famílias (Belo Horizonte) \\
\hline & \multirow{4}{*}{$\begin{array}{l}\text { Novembro } \\
\left(4^{a} \text { Onda) }\right.\end{array}$} & Testes de leitura e matemática \\
\hline & & Questionário do professor \\
\hline & & Questionário de pai ou responsável \\
\hline & & Questionário do aluno (versão reduzida) \\
\hline \multirow{4}{*}{2008} & Maio & Relatório $4^{a}$ Onda \\
\hline & \multirow{3}{*}{$\begin{array}{l}\text { Novembro } \\
\left(5^{a} \text { Onda) }\right.\end{array}$} & Testes de leitura e matemática \\
\hline & & Questionário do professor \\
\hline & & Questionário do aluno (versão ampliada) \\
\hline
\end{tabular}

Quadro 2 - Cronograma de aplicação dos instrumentos GERES, 2005-2008. Fonte: os Autores (2008). 


\section{Considerações finais}

Este artigo organiza informações básicas sobre a metodologia do GERES. Tem como objetivo ser uma referência para os pesquisadores do GERES, bem como atrair mais usuários na medida em que o banco de dados da pesquisa se torne disponível para pesquisadores externos. Não foi necessário entrar em detalhes neste artigo sobre a metodologia empregada para calcular o índice socioeconômico das famílias ou o método usado para o calculo do valor agregado das escolas. Mesmo vitais para a aplicação do modelo de análise e a compreensão dos dados, estas metodologias não foram pré-determinadas e podem mudar de acordo com os interesses dos pesquisadores.

No final do ano escolar de 2008, o GERES fará a quinta e última aplicação de instrumentos ao mesmo painel de alunos que vem acompanhando desde 2005. A partir do cálculo das proficiências dos alunos evidenciadas nestes últimos testes, a base de dados estará completa.

\section{Referências}

GOLDSTEIN, H. Methods in school effectiveness research. School Effectiveness and School Improvement, London, v. 8, n. 4, p. 369-395, 1997.

. Multilevel statistical models. 2. ed. London: Edward Arnold, 1995.

GOLDSTEIN, $\mathrm{H}$. et al. The use of value-added information in judging school performance. London: Institute of Education, 2000. Disponível em: $<\mathrm{http}$ ://www.ioe.ac.uk/hgpersonal/ Using-value-added-information.pdf>. Acesso em: 8 dez. 2008.

LEE, V. E. What are multilevel questions, and how might we explore them with quantitative methods?. Estudos em Avaliação Educacional, São Paulo, v. 24, p. $31-45,2001$.

RAUDENBUSH, S. W.; FOTIU, R. P.; CHEONG, Y. F. Inequality of access to educational resources: a national report card for eighth grade math. Educational Evaluation and Policy Analysis, Thousand Oaks, CA, v. 20, n. 4, p. 253-268, 1998.

Recebido em: 27/08/2008

Aceito para publicação em: 29/10/2008 